\title{
THÉORIE DES MODĖLES DE TEXTES APPLIQUÉE À LA TRADUCTOLOGIE
}

\begin{abstract}
Tomaszkiewicz Teresa, Théorie des modèles de textes appliquée à la traductologie [The theory of texts' models applied in the science of translation], Studia Romanica Posnaniensia, Adam Mickiewicz University Press, Poznań, vol. XXVII: 2001, pp. 163-172. ISBN 83-232-1039-X, ISSN 0137-2475.

The article describes the connection between the science of translation and contrastive textology. The foucs is on the texts of a strictly determined fixed structure, such as: instructions of use, bank or medical leaflets, recipes, job advertisements, legislative texts (a company establishment agreement, a leasing agreement, etc.), which translators may be exposed to in their professional careers. The article tries to outline these texts' models, which tend to function parallelly in different societies as a basis for their correct translation.
\end{abstract}

\section{TRADUCTOLOGIE ET LINGUISTIQUE}

Nous n'avons pas l'intention de détailler ici l'histoire des rapports entre les sciences du langage et la traductologie. Cependant un certain rappel de quelques moments importants de ces rapports nous semble nécessaire pour mieux situer la traductologie par rapport à la théorie des modèles de textes.

La traductologie dans ses débuts scientifiques, représentée par Jakobson, Nida, Mounin s'inspirait de la linguistique comparative. Les ouvrages qui se plaçaient dans cette perspective commençaient à abonder à partir de la fin des années 50 . La plupart de ces ouvrages sont consacrés à la recherche des équivalences fonctionnelles entre des formes de deux langues. Cette attitude est bien résumé par Vinay et Darbelnet (1958: 20): «...le passage d'une langue $\mathrm{A}$ à une langue $\mathrm{B}$, pour exprimer une même réalité $X$, passage que l'on dénomme habituellement traduction relève d'une discipline particulière, de nature comparative (...)». Au même moment cette vision mécaniste des langues et des traductions, où on cherchait des équivalents formels entre les formes et les structures de deux langues en contact, était renforcée par les recherches autour de la traduction automatique. Dans cette vision le problème de la contextualisation des formes était moins important.

Or, grâce à certains travaux antropologiques, par exemple, de Malinowski, Sapir, Lévi-Strauss etc. on a enrichi la réflexion traductologique par les dimensions 
culturelles et sociales. Cette nouvelle vision du processus de la traduction a été bien résumé par Pergnier (1978: 17): «Il nous semble donc nécessaire de déplacer le centre de gravité des problèmes théoriques de la traduction, des considérations sur la langue vers les considérations sur le message». Le même auteur propose de remplacer le schéma classique du processus de la traduction qui était perçu comme le passage entre

$$
\text { L.D. } \rightarrow \text { L.A. (langue de départ } \rightarrow \text { langue d'arrivée) }
$$

par le schéma:

$$
\text { M.D. } \rightarrow \text { M.A. (message de départ } \rightarrow \text { message d'arrivée). }
$$

Ainsi les traductologues ont été confrontés à des concepts comme communication, situation, contexte, message, intentionnalité etc. La réflexion traductologique à ce stade dépasse le niveau de proposition, mais elle est encore loin de traiter le texte comme unité de traduction.

\section{TRADUCTOLOGIE ET TEXTOLOGIE CONTRASTIVE}

Ce n'est qu'à la fin des années 70 que l'activité traduisante est à lier à la «textologie contrastive» (Spillner: 1981), à la détermination d'une «typologie des discours» (Reiß: 1976), d'une «typologie des textes» (Cary: 1958). La linguistique textuelle, soucieuses de l'organisation d'ensemble d'un texte, de sa progression thématique, de sa texture proprement dite (cohérence, cohésion) a aussi alimenté bien des investigations traductologiques (Neubert: 1985; Hatim et Mason: 1990, 1997; Nord: 1991) etc. On a essayé donc de déterminer des genres de textes et de catégories du discours. Ces genres et types sont déterminés par des conventions, des traditions qui nous offrent certaines normes de production et d'interprétation. Or, il faut constater que dans les différentes approches derrière les mểmes appellations se cachent parfois des notions partiellement différentes, que souvent les critères de classification sont hétérogènes et finalement qu'un même genre peut mêler divers types.

Pourtant, malgré ces difficultés dans la précision des définitions des genges de textes on en a profité dans la théorie de traduction. On peut citer ici, par exemple, Hurtado-Albir (1996), Reiß (1976), Delisle (1980).

En face de cette tendance à lier la traductologie à la textologie contrastive, on pourrait encore modifier le schéma de la traduction proposé par Pergnier en schéma suivant:

$$
\text { T.D. } \rightarrow \text { T.A. (Texte de départ } \rightarrow \text { texte d'arrivée). }
$$

A présent une nouvelle voie s'ouvre pour la traductologie. Nous pensons précisemment à la théorie des modèles des textes. Cette théorie est issue de la notion de préformé. 


\section{NOTION DE PRÉFORMÉ (FIGEMENT)}

La notion de préformé, donc d'une structure toute faite, est appliquée à trois types de phénomènes, relevant de niveaux d'analyse différents. Le premier groupe se rapporte à ce qu'on appelle aussi les idiomatismes.

\subsection{IDIOMATISMES}

Il s'agit de toute sorte de constructions comme proverbes, expressions, dictons et autres formes qui par principe se composent d'au moins deux éléments, liés entre eux d'une manière préformée. Ces structures souvent offrent des déficiences syntaxiques, lexicales ou sémantiques, tandis que leur sens global n'est pas la somme des significations de leurs composants. Ces expressions ont occupé la théorie de la traduction dans la mesure, où on constatait qu'il fallait les rendre par leurs correspondants, ayant le même sens, mais se composant souvent des unités lexicales différentes. «(...) la différence essentielle entre équivalences et correspondances: les premières s'établissent entre textes, les secondes entre les éléments linguistiques, mots, syntagmes, figements ou formes syntaxiques» (Lederer, 1994: 51).

Or, après avoir travaillé sur des corpus très divers, écrits et oraux, littéraires et non-littéraires, nous nous sommes aperçus, ce qui a été aussi confirmé par d'autres chercheurs (cf. Gülich, Krafft, 1997), que les locutions idiomatiques à proprement parler sont relativement rares dans le discours et que finalement elles ne posent pas tellement de problèmes de traduction. Il faut tout simplement savoir qu'on ne peut nullement les traduire mot à mot, ce qui n'est pas toujours évident pour les traducteurs débutants.

Pourtant n'oublions pas que les théories les plus élémentaires de la traduction distinguent souvent la traduction littéraire et la traduction dite de spécialité ou technique. Cette dernière est à leurs yeux caractérisée par une suprématie de la terminologie comparée, chaque terme scientifique de la langue source devant correspondre à un et un seul terme de la langue cible. Or, n'oublions pas que d'une part la majorité de ces termes ont aussi une structure figée, donc ces formes sont aussi à traiter dans le cadre du préformé et d'autre part dans ces textes de spécialité beaucoup plus nombreux sont des formes qu'on appelle les idiomatismes situationnels ou contextuels (Cf. Gulich, Krafft : 1997).

\subsection{IDIOMATISMES SITUATIONNELS}

Beaucoup plus fréquentes sont des expressions que l'analyse conversationnelle et l'éthnométhodologie américaine ont appelées des «idiotismes situationnels» ou des routines ou encore les expressions exprimant la politesse linguistique. Leur 
caractère préformé, au premier abord, semble moins évident. Par contre, beaucoup de chercheurs constatent qu'on voit bien leur difficulté dans les conversations, par exemple, entre le natif et le non-natif. Ces expressions doivent ễtre évidemment traduites par des équivalents les plus naturels, correspondant à la situation en question. Il est évident que pour l'expression française au téléphone: Ne quittez pas s.v.p. on va choisir un équivalent naturel en polonais: Chwileczkę, proszę poczekać. ou en fonction du contexte plus large: Proszę nie odkładać stuchawki.

Or, remarquons que dans l'optique de la traductologie ces figements appelés «idiomatismes situationnels» ou «pragmatiques» caractérisent les différents textes-types que le traducteur rencontre dans son activité quotidienne. Là, il ne s'agit pas d'une terminologie liée à un domaine particulier, mais à des formulations qui apparaissent habituellement dans un type de texte comme une lettre commerciale, une publicité, un faire-part, une offre, une lettre de candidature etc. Il est évident que leur traduction doit s'amener à la recherche d'un équivalent contextuel dans la langue d'arrivée.

\subsection{MODËLES DE TEXTES}

Enfin, la notion de préformé concerne de plus en plus «les modèles de textes» ou «les textes stéréotypés». On sait donc qu'il y a des genres de textes (écrits ou oraux ou mixtes) définis a priori, ayant des schémas conventionnels et étant un assemblage de traits situationnels, fonctionnels et structurels. Nous pensons ici aux genres tels que: faire part de décès, recette de cuisine, mode d'emploi, allocution de bienvenue, ou encore toute une série de documents que le traducteur rencontre tous les jours dans l'exécution de son métier: acte de mariage, acte notarié, catalogue d'une exposition, guide d'une ville etc. Il y en a parmi ces modèles qu'on a déjà précisemment décrits. Partout on souligne l'importance de l'existence justement d'un certain «modèle» qui détermine la façon de les rédiger. Par contre, il y en a d'autres qui n'ont pas encore trouvé une description adéquate.

Résumons-nous, le traducteur dans sa vie professionnelle rencontre beaucoup de textes dont la structure de l'ensemble est déterminé à priori. Ces genres discursifs peuvent exister dans plusieurs communautés linguistiques, ils apparaissent dans des situations comparables et ils visent des finalités communicatives similaires.

Or, ces genres discursifs peuvent avoir une structure de l'ensemble plus ou moins préformée, plus ou moins conventionnelle qui comprend un inventaire de composants textuels et de formulations préfabriquées, typiques pour le genre en question et pour une communauté linguistique déterminée.

Pourtant, certaines approches contrastives par exemple de Spillner (1981, 1983), Thiel/Thomé (1987) ou Drescher (2000), ont démontré que ces modèles sont déterminés par une certaine tradition culturelle, c'est pourquoi ils ne sont pas toujours identiques dans les différentes zones linguistiques. Pourtant la connaissance de ces matrices textuelles et des règles de leur création, nous semble 
indispensable dans le processus de traduction. Il ne s'agit pas seulement de ces expressions toutes faites, mais avant tout de la structure générale du texte, de I'organisation de ses parties constitutives, dans certains cas aussi de sa forme visuelle, de son organisation sur une feuille de papier. Tous ces éléments définissent le modèle en question, ils contribuent donc à la construction de son sens global.

Ainsi, une étude contrastive de type de textes parallèles, fonctionnant dans les différentes cultures et communautés linguistiques, permet d'établir une liste d'invariants qui caractérisent le type en question et une liste de différences qui caractérisent ce même genre dans une culture donnée, par rapport à une autre.

\section{CRITÈRES POUR LA DÉTERMINATION D'UN MODÈLE DE TEXTE}

Pour déterminer le modèle en question, nous proposons la prise en compte de facteurs suivants:

- Organisation générale de l'ensemble (parties, sous-parties, division en unités plus petites, stables).

- Forme matérielle et les éléments visuels stables (colonnes, photos, emploi des couleurs etc.).

- Type de récepteurs visées.

- Type de stratégies communicatives utilisées (informative, directionnelle, descriptive, argumentative etc.).

- Appartenance du modèle en question à un prototype textuel plus large.

- Inventaire des idiomatismes figés, typiques pour le modèle en question.

- Type de terminologies.

Ces éléments donc permettent d'une part de décrire le modèle en question et de l'autre, de voir les différences qui existent dans la réalisation de ces modèles dans les cultures et zones linguistiques différentes.

\subsection{QUELQUES MODĖLES-TYPES}

Nous avons entrepris, avec quelques groupes d'étudiants, la tentative de décrire certains modèles de cette façon-là. Il s'agit des études concernat:

- Le débat présidentiel;

- Le disours politique visant une finalité déterminée;

- Le débat télévisé traitant les problèmes culturels;

- Le talk-show;

- Certains texes en médecine légale;

- Les offres des différentes entreprises;

- Certains modes d'emploi;

- Les notices qui accompagnent les médicaments;

- Les dépliants bancaires; 
- Les publicités dans la presse et les publicités télévisées;

- La documentation technique des machines;

- Les dossiers d'adoption des enfants;

- Les catalogues des expositions d'art moderne;

- Les guides touristiques;

- Les programmes de concerts et opéras etc.

La liste reste ouverte.

Comme on voit la nature de ces discours est très variée. Il y en a dont la structure est plus figée et d'autres qui présentent plus de variantes possibles. En plus dans ces catégories, on peut déterminer des sous-catégories. Par exemple, la catégorie de dépliants bancaires peut se diviser en dépliants concernant les crédits, les ouvertures de nouveaux comptes, les cartes bancaires etc. Il n'empêche que pour chaque modèle, ou sous-modèle, on peut déterminer les caractéristiques plus ou moins figées, plus ou moins stables. Ces caractéristiques permettent de décrire le «prototype» (dans le sens de Drescher: 2000) de différents textes et de constituer des listes de formulations typiques pour ces genres.

N'oublions pas toutefois que souvent les difficultés de traduction ne résident pas tellement dans les formulations ou dans les terminologies, mais dans non correspondance des réalités qui sont mises en contact dans ce processus. Nous avons décrit ces difficultés ailleurs (Tomaszkiewicz 1998), en constatant que l'introduction du nouveau système juridique et politique en Pologne et en d'autres pays post-communistes, au début des années 1990, a augmenté le nombre des contacts économiques et autres qui ont introduit la nécessité de traduction de beaucoup de documents, entre autres juridiques. Or, justement la non correspondance des réalités, où celle polonaise était en voie de construction, a introduit beaucoup de malentendus et de problèmes d'interprétation. Nous avons cité l'exemple d'une institution qui apparaît dans l'acte de création d'une société civile ou d'une société responsabilité limitée. Il s'agit de ce qu'on appelle: Zarzq̨d Spółki. On peut le traduire en français par: direction ou administration ou gérance. Or aucun de ces termes français ne recouvre les droits et les compétences (notamment financières) dont jouit notre zarzq̨d. Les conséquences sont telles qu'un Français, ayant reconnu, dans l'acte juridique qu'il a signé, un modèle qui lui est familier (ici l'acte de création d'une société) pense y retrouver des éléments stables, typiques pour sa culture. Il ne comprend pas que là, le texte correspondant à un modèle ne correspond pas à la réalité qui lui est familière.

Résumons-nous, les membres d'une société disposent d'une compétence discursive qui leur permet de produire et de comprendre des textes correspondant à différents types ou modèles. Ayant reconnu dans un texte traduit un modèle qui leur est apparemment familier ils pensent souvent le comprendre «automatiquement». Pourtant, derrière certaines expressions ou une certaine terminologie se cachent des contenus différents, dus aux différences des réalités culturelles et sociales.

Ceci dit, nous pensons qu'une certaine textologie contrastive est nécessaire pour prévenir ou résoudre ce genre de difficultés. 


\subsection{TRADUCTEUR EN FACE DE CES MODĖLES}

Mais qu'est-ce que doit en faire un traducteur? Quelle est la voie par laquelle il doit intégrer son savoir sur les caractéristiques linguistiques et structurales d'un type de texte dans les deux cultures, pendant le processus de traduction? Bocquet (2000), dans son analyse concernant le discours économique en français, en allemand et en italien, essaie de prouver que pour pouvoir comprendre et intérioriser les caractéristiques de ce disours et saisir les différences entre ces trois cultures il faut remonter à l'époque de sa naissance. Il prouve qu'en France le discours économique est né au XVII ${ }^{\mathrm{e}}$ s. et il était fondé sur une science ancienne: la médecine. C'est pourquoi jusqu'au aujourd'hui on en retrouve des traces. Voilà quelques exemples cités par Bocquet:

Cette accumulation de paralysie ici et de frainage là explique la langueur de l'économie mondiale.

Les marchés du travail se dégradent plus vite qu'ils n'entrent en convalescence.

(Numéro spécial de Dossiers et Documents du Monde, janvier 1992: Bilan ěconomique et social)

Pays sous perfusion, le Tchad vie de l'aide publique internationale.

L'étrange maladie des caisses d'épargne

(le Monde diplomatique février 1993)

Le prix du lait amputé dans la douleur.

(L'Hebdo No 47/92)

Bocquet (2000) constate que le discours économique en Allemagne est né en prolongement de la science du droit, c'est pourquoi «les économistes allemands de la fin du $\mathrm{XX}^{\mathrm{e}}$ s. raisonnent encore en termes de loi, loi naturelle, de dérogations de sanctions. Par contre le discours économique italien est fondé sur la comptabilité commerciale générale». En conclusion, Bocquet dit que dans la traduction des textes économiques entre ces langues «la seule voie consite à maitriser suffisamment le moděle de discours de la langue cible pour le faire entrer entièrement dans les traductions quel que soit le modèle adopté par les auteurs de la langue source» (Bocquet, 2000: 26).

Mais qu'est-ce qui reste alors de la notion de fidélité à l'auteur? Jusqu'à quel point peut on se permettre l'adaptation de l'original aux exigences du moděle d'arrivée? Et quelle est la technique traductologique peut-on que doit-on adopter, par rapport aux textes dont le modèle n'existe pas dans la culture d'arrivée (par exemple le faire-part des naissances ou félicitations à l'occasion de la naissance n'existent pas en Pologne). Comment donc traduire, par un préformé, les expressions du type: Nous sommes heureux de vous féliciter de cette joie d'accueillir une belle petite fille, ou Pour le nouveau-né qui vient de faire son 
entrée dans un monde que nous lui souhaitons riche de bonheur et de satisfactions. Félicitations pour cet heureux événement etc. Et que doit faire le traducteur, quand il constate que le modèle d'arrivée prévoit une série d'informations inexistantes dans le texte de départ, mais pertinentes dans le modèle d'arrivée? Par exemple, dans beaucoup de textes modèles concernant le curriculum vitae, on introduit le point concernant la religion de la personne intéressée, ce qu'on ne trouve pas dans les modèles polonais. Ou au contraire, le modèle en question contient des informations non-pertinentes pour le modèle d'arrivée. Je peux citer ici le curriculum vitae polonais qui pendant longtemps avait un modèle difficilement acceptable en France, par exemple, avec des expressions du type: urodzitem się $w$ rodzinie robotniczej, do szkoly podstawowej uczęszczatem w Poznaniu etc. (je suis né dans une famille ouvrière, j'ai fréquenté l'école primaire à Poznań...).

\section{COMPRÉHENSION EN TRADUCTOLOGIE}

Gambier (2000) pense qu'on peut résoudre ce genre de problèmes dans la phase de compréhension par le traducteur des finalités de la traduction. Autrement dit, il doit se poser toujours et avant tout la question: Qu'est-ce qu'on lui a demandé de faire?: extraire une information, refaire un acte juridique, parvenir à un accord commercial. Ce qu'on lui a donc demandé de produire, c'est justement quelque chose, qu'il doit rendre selon certains critères d'acceptabilité.

C'est dans cette phase de compréhension, qu'il doit tenter de circonscrire:

- la situation de communication de l'original et de la traduction (l'original et la traduction ne visent pas toujours les mêmes finalités, par exemple, les textes juridiques, c'est à quoi nous revenons plus loin);

- les références exprimées par des mots ou termes mais aussi par des noms propres, des expressions culturelles;

- les marques de temps et de localisation spatiale pour établir la relation spatio-temporelle entre l'original et la traduction;

- les récepteurs du texte de départ (avec leurs connaissances présupposées, leurs attentes, leurs habitudes, leurs clichés, leur registre de langue) en les comparant avec ces caractéristiques des récepteurs potentiels de la traduction;

- les valeurs attribuées au genre de texte en question dans la culture de départ et dans la culture d'arrivée.

Gambier (2000) remarque qu'«habituellement» on détaille les stratégies ou techniques de traduction (condensation, explication, transcodage, expansion, reformulation, adaptation ponctuelle, omission, transmutation, paraphrase, compensation, emprunt, traduction littérale etc.). Par contre, rien ou presque n'est suggéré comme stratégies de lecture. Et effectivement cette lecture peut être vraiment facilitée si le traducteur l'effectue sous l'angle de la textologie contrastive. C'est celle-ci qui met en doute l'hypothèse avancée notamment par Antos (1982, cité par Drescher 2000), selon laquelle chaque texte constituerait dans sa structure de 
surface une réalisation unique. Les textes stéréotypés, qui sont souvent rencontrés par les traducteurs, suivent un schéma largement prévisible qui comprend un inventaire de composants textuels ainsi que des règles séquentielles plus ou moins contraignantes. Pour chaque composant textuel il existe généralement une série de formulations préfabriquées. Dans la production de ces textes la créativité individuelle est souvent souspendue. Cette constatation nous amène à dire que dans la traduction de ces textes la créativité textuelle du traducteur est d'autant plus souspendue. Dans une structure préétablie il existe, à notre sens, des cases vides qu'il faut compléter par des contenus plus ou moins typés.

\section{LIMITES DE LA TEXTOLOGIE CONTRASTIVE EN TRADUCTOLOGIE}

Par contre, il faut comprendre aussi que la textologie contrastive peut être utile dans la traductologie, seulement dans une certaine mesure. Comme nous avons dit plus haut, les finalités d'un texte de départ peuvent ne pas ễtre les mêmes que les finalités visées par sa traduction. Pour le récepteur indigène le texte de la Constitution de son pays est entre autres un inventaire des normes obligatoires pour lui. Pour les récepteurs de sa traduction, c'est un texte informatif, qui renseigne sur les normes obligatoires pour les citoyens d'un autre pays. Pour le récepteur de l'original le texte du code civil est obligatoire. Sa traduction devient obligatoire pour le récepteur étranger, quand celui-ci conclut un acte légal où il devient partie dans une affaire juridique. L'acte de naissance est un document qui dans le contexte de départ est utilisé dans certains contextes juridiques, sa traduction aura probablement les mêmes finalités dans la culture d'arrivée. Les films engagés politiquement, comme par exemple «L'homme de fer» de Wajda, visaient des finalités différentes en Pologne, remplissant le rôle presque d'une manifestation politique, mais dans les traductions en français ou en anglais ils ont acquis beaucoup plus la fonction informative.

Nous pensons donc que de même qu'on s'est mis d'accord que la linguistique contrastive n'est qu'une étape pour parvenir à la traduction, la textologie contrastive peut donner seulement certains outils à la traduction qui est une production de textes à base du modèle de départ en intégrant, dans la mesure du possible, les caractéristiques du modèle d'arrivée. Pourtant, souvent il ne s'agit pas du même modèle dans les deux cultures.

Un ensemble de recherches actuelles, centrées sur des corpus des textes traduits, confirme que la traduction induit une langue hybride, qui n'est pas la résultane d'interférences, de calques... Ces traits sont spécifiques parce qu'ils ne sont pas ordinairement présents en quantité dans les textes rédigés directement en langue d'arrivée. L'analyse empirique de divers corpus, notamment à Manchester, depuis 1993 (avec M. Baker, S. Laviosa et autres) confirme que ces traits traductionnels apparaissent indépendemment des langues de départ. 
Ceci dit, l'activité traduisante est une médiation entre les modèles de textes et elle contribue de sa part aussi à la textologie contrastive, mais elle n'est pas à confondre avec celle-ci.

\section{BIBLIOGRAPHIE}

Antos G. (1982), Grundlagen einer Theorie des Formulierens. Textherstellung in geschriebener und gesprochener Sprache. Tübingen.

B ocquet C. (2000), La notion de modèle de discours appliquée à la traduction, Studia Romanica Posnaniensia XXV/XXVI, pp. 21-28.

Cary E. (1958), Comment faut-il traduire?, Lille: Presses Universitaires de Lille.

Drescher M. (2000), Pour une analyse contrastive des types de discours: L'exemple du faire-part de dèces en français et en espagnol, Studia Romanica Posnaniensia XXV/XXVI, pp. 67-80.

Delisle J. (1980), L'analyse du discours comme méthode de traduction, Ottawa: Editions de l'Université d'Ottawa (2e éd.).

Gambier Y. (2000), Traduction et analyses de discours: typologie croisée, Studia Romanica Posnaniensia XXV/XXVI, pp. 97-108.

Gülich E., Krafft U. (1997), Le rôle du 'préfabriqué' dans le processus de production discursive, in: Michael Martins-Baltazar, La locution entre langue et usages. Fontenay-aux-Roses: ENS - Editions Fontenay/Saint-Cloud, pp. 241-276.

Hatim B. et Mason I. (1990), Discourse and the Translator, London, New York: Longman.

Hatim B. et Mason I. (1997), Communication across cultures: Translation and Contrastive Text Lingustics, Exeter: Exeter U.P.

Hurtado-Albir A. (1996), La traduction: classification et éléments d'analyse, Meta 41(3), pp. 366-377.

Lederer M., (1994), La traduction aujourd'hui, Paris: Hachette.

Neubert A. (1985), Text and translation, Leipzig: VEB Verlag Enzyklopädie.

Nord C. (1991), Text Analysis in Translation. Amsterdam, Atlanta, GA: Rodopi.

Pergnier M. (1993) ( $\mathrm{I}^{\mathrm{c}}$ éd. 1978), Les fondements socio-linguistiques de la traduction, Presses Universitaires de Lille.

Reiß C. (1976), Texttyp und Übersetzungsmethode. Der Operative Text. Kronberg: Scriptor.

Spillner B. (1981), Textsorten im Sprachvergleich. Ansätze zu einer kontrastiven Textologie in: Kühlwein W., Thome G., Wilss W. (éds), Kontrastive Linguistik und Übersetzungswissenschaft. München, pp. 239-250.

Spillner B. (1983), Zur kontrastiven Analyse von Fachtexten - am Beispiel der Syntax von Wetterberichten, LiLi. Zeitschrift für Literaturwissenschaft und Linguistik, 13, Bielefeld, pp. 110-123.

Thiel G., Thomé G. (1987), Resolutionen. Ein empirisch enwickelter Beitrag zur Textanalyse. Tübingen.

Tomaszkiewicz T. (1998), Traduction juridique en Pologne: affrontement de deux mondes distincts, in: Balard, M. (éd.) Europe et Traduction, Arras: Artois Presses Université, Les Presses de l'Université d'Ottawa, pp. 283-294.

Vinay J.P., Darbelnet J. (1958), Stylistique comparée du français et de l'anglais. Paris: Marcel Didier. 